\title{
Molecular Motions in Chitosan Studied by Dielectric Relaxation Spectroscopy
}

\author{
M. T. Viciosa, ${ }^{\dagger}$ M. Dionísio, ${ }^{*, \dagger}$ R. M. Silva, ${ }^{\ddagger} \S$ R. L. Reis, ${ }^{\ddagger}, \S$ and J. F. Mano ${ }^{\ddagger \S}$ \\ REQUIMTE/CQFB, Departamento de Química, FCT, Universidade Nova de Lisboa, 2829-516 Caparica, \\ Portugal, Department of Polymer Engineering, University of Minho, Campus de Azurem 4800-058 \\ Guimarães, Portugal, and 3B's Research Group-Biomaterials, Biodegradables, Biomimetics, \\ Department of Polymer Engineering, University of Minho, Campus de Gualtar 4710-053 Braga, Portugal
}

Received May 28, 2004

\begin{abstract}
Neutralized and nonneutralized chitosan films subject to different thermal treatments were studied by dielectric relaxation spectroscopy from -130 to $+150{ }^{\circ} \mathrm{C}$ in the frequency range between $20 \mathrm{~Hz}$ and $1 \mathrm{MHz}$. Two main relaxation processes, both arrhenian type, were detected: process I at temperatures below $0{ }^{\circ} \mathrm{C}$ with a mean activation energy of $49 \pm 1 \mathrm{~kJ} \mathrm{~mol}^{-1}$, which has the characteristics of a secondary relaxation process related with local chain dynamics, and process II observable at higher temperatures with an activation energy of $94 \pm 2 \mathrm{~kJ} \mathrm{~mol}^{-1}$, correlated with dc conductivity, which is found in dried polysaccharides systems. Process I is always observed in neutralized chitosan, but it is strongly depleted in the wet nonneutralized form. Although the location of process I is independent of $\mathrm{NH}_{2} / \mathrm{NH}_{3}{ }^{+}$side group, process II deviates to higher temperatures with dryness in both chitosan forms, being located at lower temperatures in nonneutralized chitosan.
\end{abstract}

\section{Introduction}

After cellulose, chitin is the second most abundant natural polymer. It is associated with the biomineralization of the exoskeleton of crustaceans, molluscs, insects, and other invertebrates and appears also in the cells of fungi and moulds. ${ }^{1}$ Chitosan is the partially deacetylated product of chitin. As chitin cannot be processed by melt-based techniques, the possibility of dissolving chitosan enables it to be shaped into membranes or fibers (by solvent casting or wet spinning), making this chitin-based material very attractive for a variety of applications. After processing, chitosan may revert to chitin though an acetilation reaction. Lazaridou and Biliaderis ${ }^{2}$ reported a wide range of potential uses of chitosan in different fields, such as environmental (prevention of water pollution), nutrition (flavor encapsulation, recovery and removal of undesirable compounds, seed coatings), or biomedical applications (against hypertension, antimicrobial and hypocholesterolemic activity and in controlled delivery of bioactive agents).

It is thus of great interest to fully characterize the solidstate physical and physicochemical properties of chitosan, including the molecular mobility that occurs within the polymeric structure, in both wide temperature and time scale ranges. As chitosan owns an interesting collection of dipolar groups, dielectric relaxation spectroscopy (DRS) may be a suitable technique to probe translational/rotational molecular motions, as it enables one, with high sensitivity, to look into

\footnotetext{
* To whom correspondence should be addressed. E-mail: madalena.dionisio@dq.fct.unl.pt.

Universidade Nova de Lisboa.

Department of Polymer Engineering, University of Minho.

$\S 3$ B's Research Group, University of Minho.
}

dipolar fluctuations in broad temperature and frequency ranges (up to 16 decades). DRS studies on different polysaccharides have been reported in the literature, mainly for cellulose $^{3-5}$ and for starch. ${ }^{6,7}$ Einfeldt et al ${ }^{8,9}$ compiled a huge amount of dielectric results of a variety of polysaccharides, having found a common pattern for the detected relaxation processes. At low temperatures, the local side-group motions in the repeating unit originate a $\gamma$-relaxation. Still in the lowtemperature range $\left(-135\right.$ to $\left.+20{ }^{\circ} \mathrm{C}\right)$, a $\beta$-relaxation emerges, attributed to local main chain motions, due mainly to fluctuations within the glycosidic bonds. This process may own some degree of cooperativity, which does not occur in the $\gamma$-relaxation. ${ }^{4}$ In wet samples in the room-temperature range, a $\beta_{\text {wet }}$ relaxation is observed that could be probably assigned to orientational motions of a mixed phase of both polysaccharide and water (or other swelling solvents), which is formed in wet systems by a swelling process, ${ }^{9}$ although its molecular origin is not well established. At higher temperatures $\left(80-180{ }^{\circ} \mathrm{C}\right)$, a $\sigma$-process emerges, due to proton migration. Specifically, in chitosan, Nogales et al. ${ }^{10}$ report two relaxation processes: (i) a local relaxation, with similar characteristics with the abovementioned $\beta$-relaxation, ${ }^{8,9}$ but attributed to hydrogen-bonded water, and (ii) a conduction process related to water molecules which becomes desorbed upon heating at $T>80{ }^{\circ} \mathrm{C}$ and that disappears after water desorption at high temperatures.

Thus, DRS studies on chitosan are scarce and ambiguous, despite the scientific and technologic importance of this biopolymer. It is the purpose of this work to bring some insight in the molecular origin of the observed relaxation processes.

The degree of deacetylation (DD), corresponding to the molar ratio of GlcN units in the copolymer (see Scheme 1) 
Scheme 1. Acid-Base Equilibrium of Chitosan
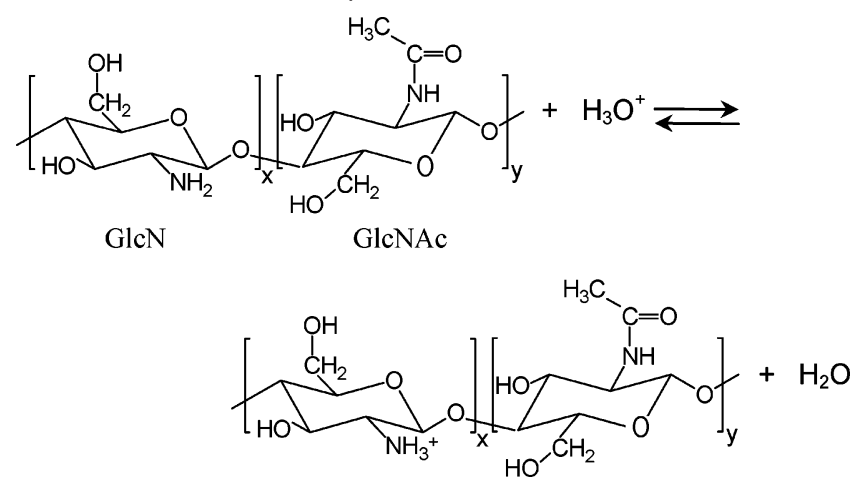

has a striking effect on chitosan's physical and chemical properties. Therefore, samples with a specific degree of deacetylation $(70 \pm 5) \%$ were studied. As chitosan films are usually prepared by solvent casting, the final material appears highly protonated. Such films are highly sensitive to water (they present low stiffness and resistance in the presence of water), and a neutralization with alkaline solutions is needed to give them mechanical integrity and insolubility. Both nonneutralized and neutralized chitosan films subject to different thermal treatments will be studied by DRS, to detect any change upon the molecular mobility with the charge in the free amino group of chitosan and to evaluate the influence of the water content.

\section{Experimental Section}

2.1. Chitosan Films Preparation and Characterization. Chitosan is a cationic heteropolysaccharide composed of $\beta$-(1,4)-2-deoxy-2-amino-D-glucopyranose $(\mathrm{GlcN})$ and $\beta$ $(1,4)$-2-deoxy-2-acetamido-D-glucopyranose (GlcNAc) units (Scheme 1).

Chitosan from crab shells was purchased from SigmaAldrich, ref. A44,887-7. Its degree of deacetylation (DD) was determined by Fourier transformed infrared spectroscopy (FTIR), following the methods described by Baxter et al., ${ }^{11}$ method I, and Brugnerotto et al., ${ }^{12}$ method II.

Method I is based on the measurement of absorption bands height at $1655\left(A_{1655}\right)$ and $3450\left(A_{3450}\right)$, using the following equation:

$$
\mathrm{DD}(\%)=100-\left(A_{1655} / A_{3450}\right) \times 115
$$

Method II makes use of the absorption ratio $A_{1320} / A_{1420}$, being the DD determined according to the following equation:

$$
100-\mathrm{DD}(\%)=31.92 \times A_{1320} / A_{1420}-12.20
$$

According methods I and II, the degree of deacetylation was found to be, respectively, $68( \pm 2) \%$ and $70( \pm 5) \%$ calculated from three different spectra.

The viscosity average molecular weight, was determined according Terbojevich and Cosani, ${ }^{13}$ as $M_{\mathrm{v}}=594 \mathrm{kDa}$. In acidic conditions, the free amino groups can be protonated (making chitosan a polyelectrolyte in dilute aqueous solutions of several acids ${ }^{14}$ ), the degree of protonation determinig its solubility. ${ }^{15}$ A solution was prepared by dissolving chitosan $(1 \mathrm{wt} \%)$ in acetic acid solution (1wt \%). The membranes were prepared by solvent casting, by pouring the solution into Petri dishes followed by dryness at $37{ }^{\circ} \mathrm{C}$. The membranes obtained by this procedure are in the nonneutralized form with the amino side group protonated. To obtain the neutralized films (neutral amino side groups), the membranes were further neutralized in $\mathrm{NaOH}(0.1 \mathrm{M}$ solution) for $10 \mathrm{~min}$, washed thoroughly with distilled water, and re-dried at $37^{\circ} \mathrm{C}$.

In the present work, we studied both neutralized and nonneutralized chitosan films, using the following designations: wet, samples that were measured after preparation with no further drying or thermal treatment, according to thermogravimetric experiments (see section 3.1), the water content in these samples is $13.5 \pm 0.5 \%$; annealed, previous wet samples after being subject to annealing at $150{ }^{\circ} \mathrm{C}$ for 20 min.

Duplicate annealed samples of each type were analyzed.

2.2. Thermogravimetry. Thermogravimetry experiments (TGA) were performed in a Perkin-Elmer TGA apparatus, using high purity nitrogen as a purge gas. Both neutralized and nonneutralized chitosan, with initial weight of $\sim 3 \mathrm{mg}$, and kept in the same environment (ca. 50\% humidity, at room temperature, as measured by a laboratory hygrometer), were studied under the following temperature program: heating at $5{ }^{\circ} \mathrm{C} / \mathrm{min}$ from 30 to $150^{\circ} \mathrm{C}$, followed by a cooling stage at $20^{\circ} \mathrm{C} / \mathrm{min}$ down to $\sim 35{ }^{\circ} \mathrm{C}$, and finally by a second heating step up to $150{ }^{\circ} \mathrm{C}$, at $5{ }^{\circ} \mathrm{C} / \mathrm{min}$.

2.3. Dielectric Relaxation Spectroscopy. The dielectric measurements were carried out using a Hewlett-Packard impedance analyzer HP 4284A, covering a frequency range from $20 \mathrm{~Hz}$ to $1 \mathrm{MHz}$. The temperature control was assured by the Quatro Cryosystem from Novocontrol GmbH. The chitosan samples were placed between two gold plated electrodes (diameter $20 \mathrm{~mm}$ ) of a parallel plate capacitor. The sample cell (BDS 1200) was mounted on a cryostat (BDS 1100) and exposed to a heated gas stream being evaporated from a liquid nitrogen dewar. The temperature control is performed within $\pm 0.05{ }^{\circ} \mathrm{C}$. The data are given in terms of the frequency dependence of the complex permittivity: $\epsilon^{*}(\omega)=\epsilon^{\prime}(\omega)-i \epsilon^{\prime \prime}(\omega)$, with $i^{2}=-1$.

2.3.1. Fitting Function. The Havriliak-Negami (HN) model function ${ }^{16}$ was used for the data analysis. In the frequency domain, the HN function reads

$$
\epsilon^{*}=\epsilon_{\infty}+\sum_{j} \frac{\Delta \epsilon_{j}}{\left[1+\left(i \omega / \omega_{0 j}\right)^{\alpha_{j}}\right]^{\beta_{j}}}-i \frac{\sigma}{2 \pi \epsilon_{0} f^{c}}
$$

where $j$ is the number of relaxation processes and $\omega_{0}=2 \pi$ $f_{0}$ is the characteristic $\mathrm{HN}$ frequency closely related with $f_{\max }$, the frequency of maximal loss. From this frequency a characteristic relaxation time $\tau=1 /\left(2 \pi f_{\max }\right)$ can be obtained; $\epsilon_{\infty}$ is the real permittivity for high frequencies compared to $f_{\max }$, i.e., $\epsilon_{\infty} \cong \epsilon^{\prime}\left(f \gg \mathrm{f}_{\max }\right) ; \Delta \epsilon$ is the dielectric intensity or relaxation strength, measuring the difference of the real permittivity at low and high frequencies with respect to $f_{\max }$. The curve shape parameters $\alpha$ and $\beta(0<\alpha<1,0<\alpha \beta<$ 1) describe the slopes of the $\epsilon^{\prime \prime}$ curve below and above the frequency of the peak: $\alpha=\partial \log \epsilon^{\prime \prime} / \partial \log f$, for $f \ll f_{0}$, and $\alpha \beta=-\partial \log \epsilon^{\prime \prime} / \partial \log f$ for $f \gg f_{0}$; the $\alpha$ value is related 


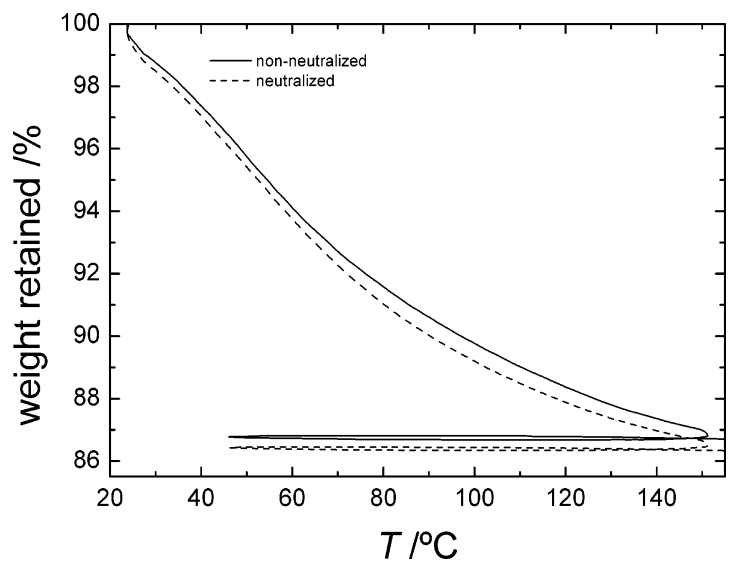

Figure 1. Thermogravimetry results on nonneutralized (solid line) and neutralized (dashed line) chitosan membranes (initial weight within $3.0 \pm 0.1 \mathrm{mg}$ ). The temperature program comprises a first heating at $5{ }^{\circ} \mathrm{C} / \mathrm{min}$, a cooling at $20^{\circ} \mathrm{C} / \mathrm{min}$ and a second heating at $5^{\circ} \mathrm{C} / \mathrm{min}$.

with the broadness of the relaxation while $\beta$ describes its asymmetry (Debye behavior is given by $\alpha=\alpha \beta=1$ ).

The dielectric data in the frequency domain were fitted by eq 1 , and the fitting parameters are $\Delta \epsilon, \alpha, \beta$, and $\tau$. The conductivity contribution for the dielectric loss was described by the second term of eq 1 , where $\epsilon_{0}$ is the vacuum permittivity; $\sigma$ and $c$ are fitting parameters; $\sigma$ is related to the dc conductivity of the sample and $c$ describes the broadening of the relaxation time distribution for the $\mathrm{dc}$ conductivity.

\section{Results and Discussion}

3.1. Thermogravimetry Results. Figure 1 shows the thermogravimetry results on both neutralized and nonneutralized chitosan. The amount of free-water may be evaluated by the decrease of sample weight during the first heating scan. Very similar water content was found for the two materials: $13.5 \pm 0.5 \%$. A second heating run confirmed that the first heating was quite efficient in terms of freewater elimination as at the end of the second heating processes the weights of both samples are found to be very similar. Therefore, one may consider that $150{ }^{\circ} \mathrm{C}$ should be a good compromise temperature to remove water in chitosan, being low enough to prevent thermal degradation of the materials. It should be noticed that, as in cellulose, ${ }^{3}$ some adsorbed water might still exist in the chitosan structure, even for such annealing at elevated temperature.

3.2. Dielectric Results. 3.2.1. Wet Samples. The dielectric spectra of both neutralized and nonneutralized wet chitosan samples (see the Experimental Section) were collected in ascending temperature steps starting from $-120^{\circ} \mathrm{C}$ up to $+150{ }^{\circ} \mathrm{C}$. A relaxation process was detected in the lowtemperature range, between -120 and $0{ }^{\circ} \mathrm{C}$, in the neutralized form. This process is much less intense and ill defined in nonneutralized chitosan. At higher temperatures, the results are influenced by very high conductivity values. Nevertheless in a logarithmic plot of the dielectric loss, it is possible to detect a relaxation process starting from around $-10^{\circ} \mathrm{C}$.

In Figure 2, the dielectric loss factor at $1 \mathrm{kHz}$, obtained from the isothermal measurements, is plotted in function of

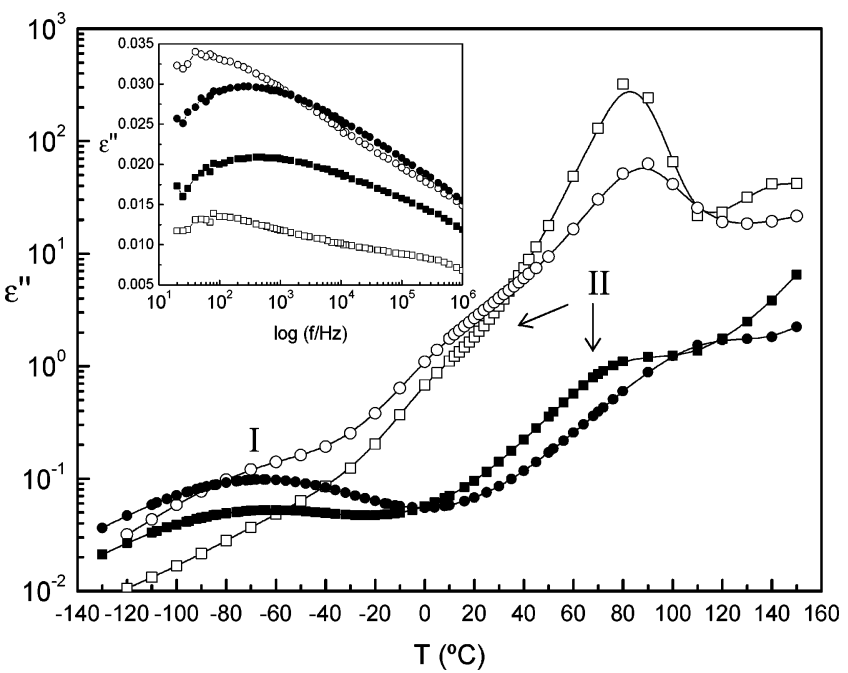

Figure 2. Loss factor at $1 \mathrm{kHz}$ in function of temperature for chitosan (a) neutralized and (b) nonneutralized: open symbols, wet samples; full symbols, annealed samples; circles, neutralized; squares, nonneutralized. Inset: dielectric loss at $-90^{\circ} \mathrm{C}$.

temperature for these wet samples (open symbols), in a logarithmic scale to enhance the relaxation processes and to cover all data since they vary by several orders of magnitude.

The changes in slope of the $\epsilon$ " representation denounce a low-temperature process for neutralized chitosan (circles) and another relaxation process at temperatures from $-10{ }^{\circ} \mathrm{C}$ to around $50{ }^{\circ} \mathrm{C}$ that occurs in both samples. Additionally, the dielectric loss factor of both neutralized and nonneutralized (squares) chitosan presents a maximum at, respectively, 90 and $80{ }^{\circ} \mathrm{C}$, which location is invariant with the frequency.

The further decrease of conductivity at higher temperatures is due to a loss of water by evaporation. It is interesting to verify that both the maximum of the conductivity peak related with the water release and the onset of conductivity occur earlier, in the temperature scale, in the nonneutralized sample. Additionally, the conductivity values are higher in this form (higher $\epsilon_{1 \mathrm{kHz}}^{\prime \prime}$ values for $T>50^{\circ} \mathrm{C}$ ). Since the TGA analysis revealed similar water contents in both chitosan forms $(13.5 \%)$, this observation could indicate either a higher mobility and/or number of conducting species namely the acetate counteranions of the $\mathrm{NH}_{3}{ }^{+}$groups in nonneutralized chitosan.

3.2.2. Annealed Samples. After these first series of measurements, the wet samples were annealed $20 \mathrm{~min}$ at 150 ${ }^{\circ} \mathrm{C}$ and remeasured isothermally in descending temperature steps starting from $+150{ }^{\circ} \mathrm{C}$ down to $-130^{\circ} \mathrm{C}$. The $\epsilon^{\prime \prime}$ plots at $1 \mathrm{kHz}$, obtained from isothermal measurements, of both neutralized and nonneutralized annealed samples were included in Figure 2 (full symbols). Two relaxation processes were detected in the entire temperature range. One was located at temperatures below $0{ }^{\circ} \mathrm{C}$ (named process I), which was previously noticed in the wet forms, and a second process was located at temperatures above $20{ }^{\circ} \mathrm{C}$ (named process II), which was also observed in the wet samples but highly masked by the water release.

The intensity of process I enhances after the annealing, mainly for nonneutralized chitosan. Otherwise stated, with the increase of water content, the process almost vanishes in this chitosan form. This fact may indicate that the $\mathrm{NH}_{3}{ }^{+}$ 
side groups of nonneutralized chitosan are strongly involved in hydrogen bonds with water, relative to the $\mathrm{NH}_{2}$ groups of the neutralized form, depleting the relaxation process. The higher hydrogen bond interaction energy of the cationic $-\mathrm{NH}_{3}{ }^{+}$probe is confirmed in computational studies in biological probes able to form multiple hydrogen bonds. ${ }^{17,18}$ Therefore, an enhanced interaction in wet nonneutralized chitosan/water system via hydrogen bonding, together with an increased water retention capacity could weaken the lowtemperature process. With the further removal of water, achieved by the annealing at $150{ }^{\circ} \mathrm{C}$, the dielectric strength increases. In the inset of Figure 2, the large increase of dielectric loss and the definition of the relaxation peak after annealing are clear, in nonneutralized chitosan at $-90{ }^{\circ} \mathrm{C}$; the relaxation process is also enhanced in neutralized chitosan despite the low-frequency tail in the first run due to the second process located at higher temperatures.

Each different type of relaxation process (I and II) will be analyzed in more detail in the following sections.

3.3. Low-Temperature Process (Process I). Figure 3 shows the relaxation process of annealed chitosan located at the lowest temperatures in a representation of $\epsilon^{\prime \prime}$ as a function of frequency ( $\mathrm{a}$ and $\mathrm{b}$, respectively, for neutralized and nonneutralized chitosan).
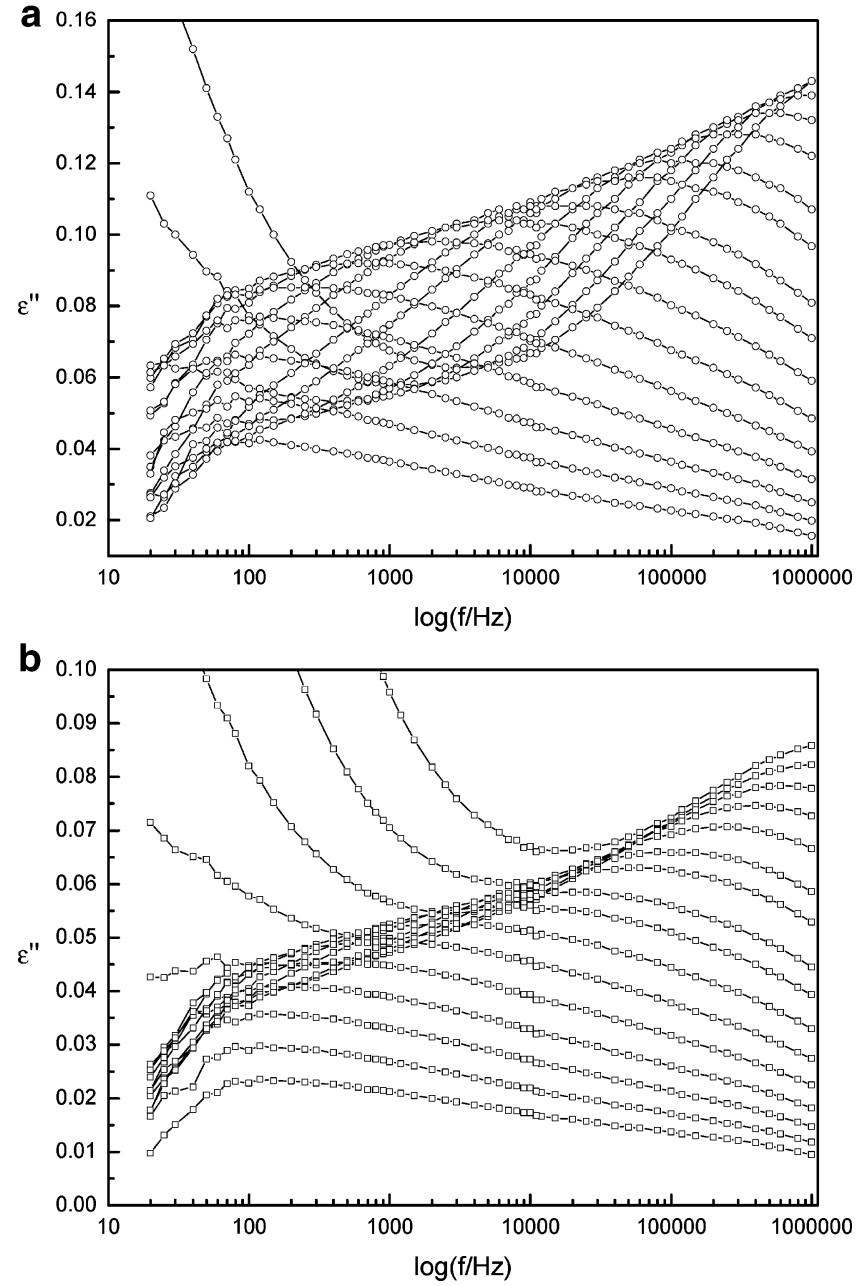

Figure 3. Loss curves in the temperature region between -120 and $0{ }^{\circ} \mathrm{C}$ in steps of $10^{\circ} \mathrm{C}$ for annealed chitosan (a) neutralized and (b) nonneutralized.

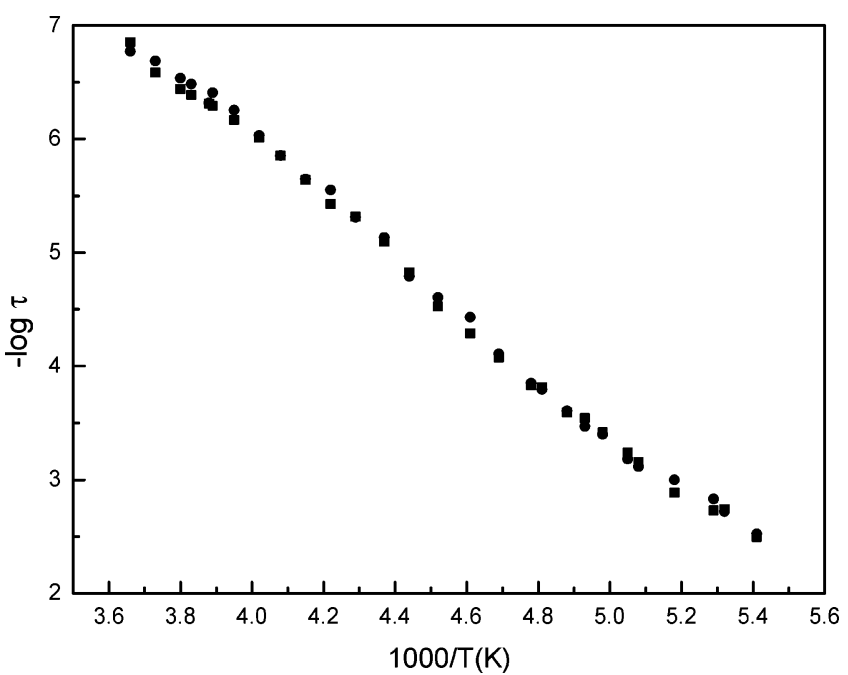

Figure 4. Arrhenian plots for the low-temperature relaxation process in annealed samples: circles, neutralized; squares, nonneutralized. The slight change on the slope at $1000 / T 4.4$ is due to the incoming of process II; all of the data were considered in the calculation of the activation energy.

The features of this low-temperature relaxation visible below $0{ }^{\circ} \mathrm{C}$ are similar for neutralized and nonneutralized annealed chitosan. In addition, the loss curves of the nonneutralized annealed chitosan present a low-frequency tail (see Figure 3b), due to the closeness of the hightemperature relaxation process (process II). This increasing of $\epsilon^{\prime \prime}$ for nonneutralized chitosan was visible in Figure 2 at $1 \mathrm{kHz}$ for temperatures higher than $-20{ }^{\circ} \mathrm{C}$; in neutralized chitosan, processes I and II are more separated in the temperature range; thus, such an increase is only verified at higher temperatures (near $0{ }^{\circ} \mathrm{C}$ ).

The influence of process II reflects in the fitting procedure of this low-temperature process. Therefore, there is a critical temperature, for each chitosan sample, above which two HN functions are necessary to obtain a good fit of the data ( -44 ${ }^{\circ} \mathrm{C}$ for nonneutralized chitosan and $-28{ }^{\circ} \mathrm{C}$ for the neutralized sample). Bellow this critical temperature, the shape of $\epsilon^{\prime \prime}$ curves changes to a broader contour which could be fitted by only one HN function. This behavior reflects in the shape parameters, whereas the $\beta$ fitting parameter has a value of $0.94 \pm 0.10$ at the highest temperatures when $2 \mathrm{HN}$ functions are used, denoting a relatively symmetrical process, bellow the critical temperature the $\beta$ values decrease to $0.67 \pm 0.10$. At the same time, the $\alpha$ fitting parameter shows a slight decrease from around $0.33 \pm 0.03$ to $0.28 \pm 0.02$; these low $\alpha$ values indicate a broad distribution of relaxation times. The dielectric strength, a fitting parameter in $\mathrm{HN}$ function, is almost invariant with the temperature for both samples, being $1.00 \pm 0.05$ and $0.56 \pm 0.05$, respectively, for neutralized and non-neutralized chitosan, in the temperature range of -88 to $0{ }^{\circ} \mathrm{C}$.

Figure 4 shows the Arrhenius plot for process I of both annealed neutralized (full circles) and nonneutralized samples (full squares).

There is some inflection bellow 4.4 (in the 1000/T scale) due to the incoming of process II. As previously mentioned, this reflects in the fitting procedure where $1 \mathrm{HN}$ fitting function is replaced by $2 \mathrm{HN}$ functions. Nevertheless, the 
slight change of slope observed does not alter significantly the activation energy and thus, the entire range of temperature was considered $\left(r^{2}=0.99\right)$. Additionally, both samples present nearly the same activation plot, yielding a mean activation energy of $49 \pm 1 \mathrm{~kJ} \mathrm{~mol}^{-1}$. The preexponential factor, $\tau_{0}$, is $(4.4 \pm 0.7) \times 10^{-17} \mathrm{~s}$. This activation energy value agrees very well with $47.8 \mathrm{~kJ} \mathrm{~mol}^{-1}$ disclosed by Einfeldt et al. ${ }^{9}$ for chitosan.

This sub- $T_{\mathrm{g}}$ process is usually observed in polysaccharide materials. Different molecular origins for this relaxation process have been suggested in the literature that are summarized and discussed by Einfeldt et al. ${ }^{9}$ From the dielectric results in a series of polysaccharides, the authors deduce that this process represents a local main chain motion being, therefore, a $\beta$-type process, which dynamics is mainly influenced by the glycosidic linkage.

Secondary relaxations, namely the $\beta$ relaxation in polymers, are characterized by their broadness ( $\alpha$ shape parameter under 0.5 ) and arrhenian behavior. Therefore, the low values of the $\alpha$ shape parameter $(0.28-0.33)$ and the activation energy obtained for process I in the present work, are coherent with a secondary relaxation, thus with localized mobility. Nevertheless the differences obtained between neutralized and nonneutralized wet samples and the intensity differences even after annealing, seem to indicate that additionally to the type of glycosidic linkage, the dynamics of this $\beta$ process is also influenced by the amine side group, namely by its protonation state that determines the strength of the interaction with water.

3.4. High-Temperature Process (Process II). Figure 5 presents the loss curves for the high-temperature process, process II, between 10 and $150{ }^{\circ} \mathrm{C}$ for (a) neutralized and (b) nonneutralized annealed chitosan.

The location of process II in nonneutralized chitosan is deviated to higher frequencies relative to the neutralized chitosan, indicating a higher mobility of the relaxation process in the nonneutralized condition. This effect is also visible in the $\epsilon^{\prime \prime}$ plot presented above in Figure 2, where the peak for the nonneutralized chitosan appears earlier in the temperature scale.

The shape parameters of process II are $\beta=0.90 \pm 0.10$ and $\alpha=0.68 \pm 0.02$ for both neutralized and nonneutralized samples and are almost temperature independent.

The activation plot of this high-temperature process is presented in Figure 6.The plots are Arrhenian type, with the same slope for both neutralized and nonneutralized chitosan, yielding a mean activation energy of $94 \pm 2 \mathrm{~kJ} \mathrm{~mol}^{-1}$. The difference is found in the preexponential factor, which is 2 $\times 10^{-17} \mathrm{~s}$ for the neutralized and $7 \times 10^{-18} \mathrm{~s}$ for nonneutralized chitosan. According to Eyring ${ }^{19}$ formalism, $\tau_{\mathrm{o}}$ values lower than the Debye time $\left(\approx 10^{-13} \mathrm{~s}\right)$, imply a positive activation entropy $\left(\Delta S^{\ddagger}>0\right)$; therefore, the $\tau_{0}$ values here obtained reflect an activation entropy effect which is higher in the nonneutralized annealed sample, meaning that the mobility of process II is more complex in this sample relative to the neutralized one.

As previously mentioned (see Introduction), Einfeldt ${ }^{9}$ detected a $\beta$-wet process in wet cellulose-like materials near room temperature and Nogales et al. ${ }^{10}$ detected in chitosan
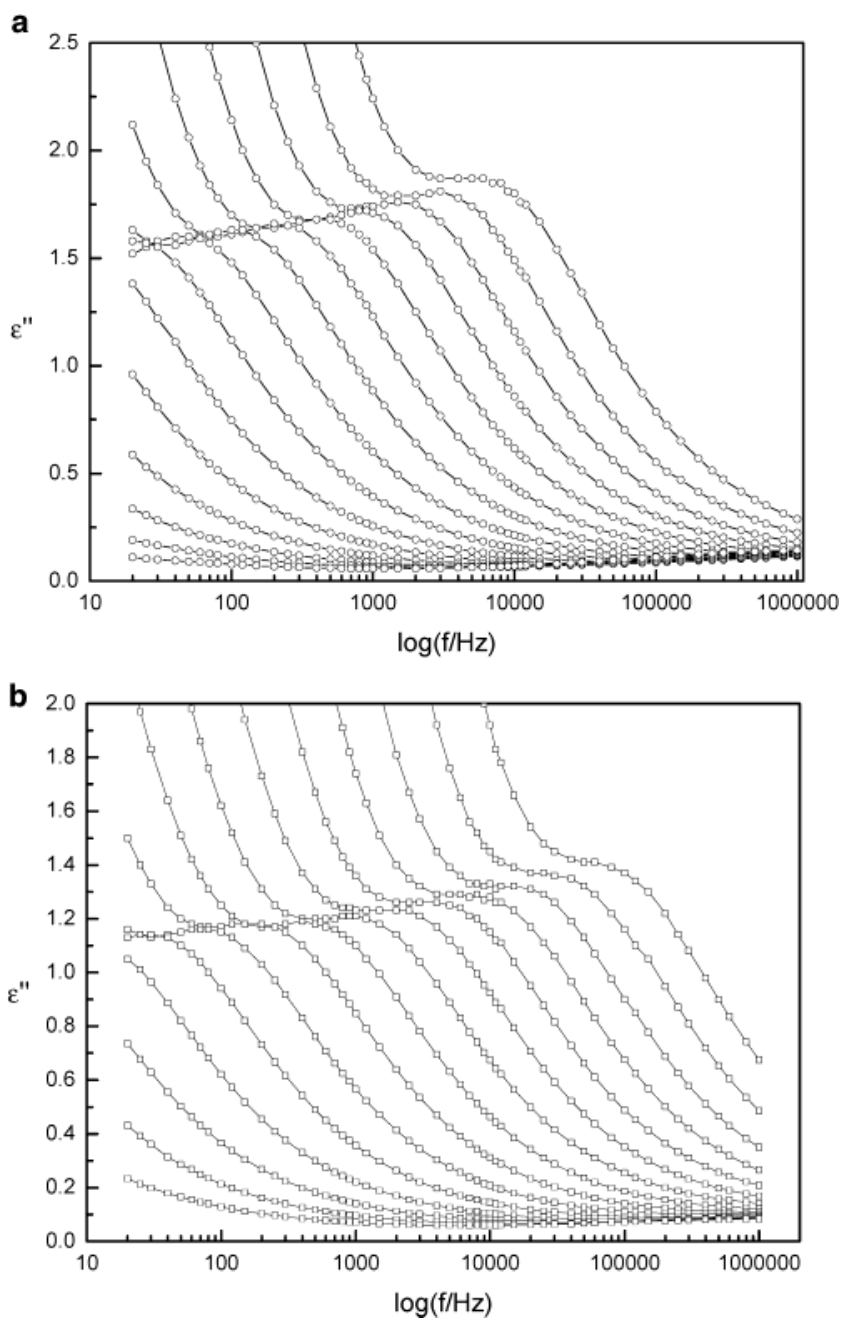

Figure 5. Loss curves of annealed chitosan in the temperature region between 10 and $150{ }^{\circ} \mathrm{C}$ in steps of $10^{\circ} \mathrm{C}$; (a) neutralized and (b) nonneutralized.

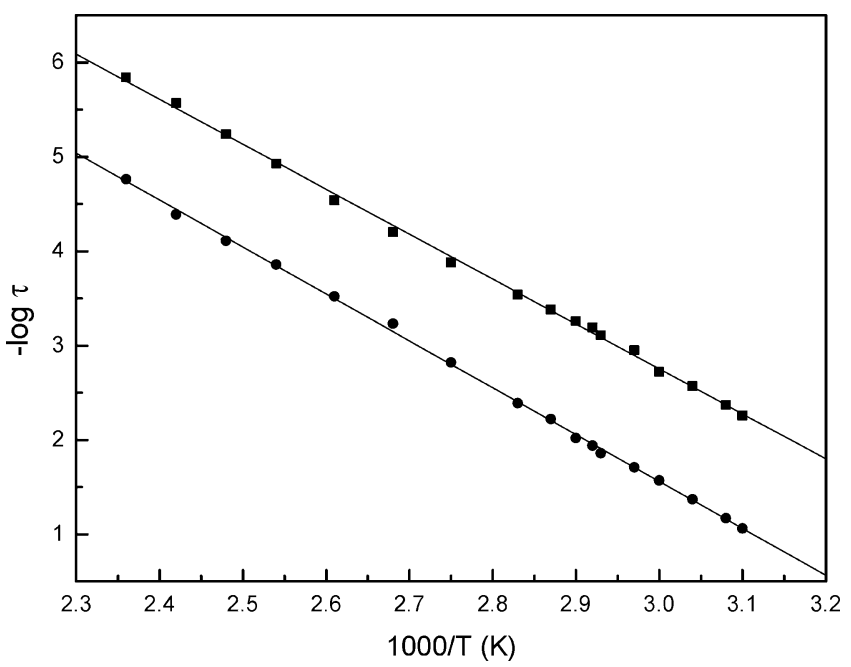

Figure 6. Arrhenian plots for process II for annealed chitosan: circles, neutralized; squares, nonneutralized.

a process related with water desorption that vanishes after water removal. The fact that our process II is still observed in annealed samples, i.e., after water removal, pulls apart the interpretation as a $\beta$-wet relaxation. Rather, the features of the present process II resemble the $\sigma$ process found in a series of polysaccharides, ${ }^{8}$ namely, the high activation energy 


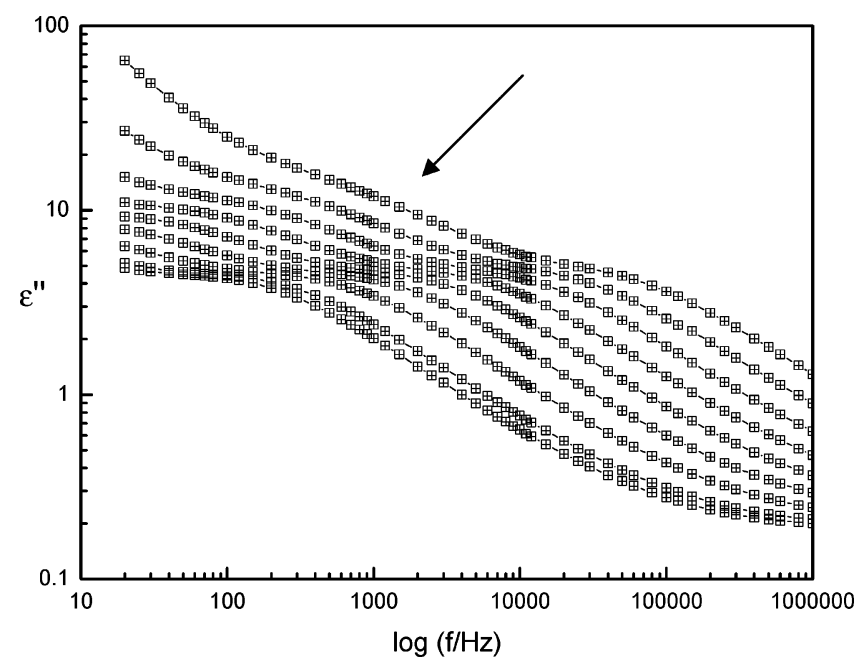

Figure 7. Dielectric loss spectra of an annealed nonneutralized chitosan sample (previously dried $48 \mathrm{~h}$ at $140{ }^{\circ} \mathrm{C}$ in a vacuum) showing the MWS effect at high temperatures in the low-frequency side of process II (arrow).

value here found, $94 \pm 2 \mathrm{~kJ} \mathrm{~mol}^{-1}$, that is in agreement with the value of $95-110 \mathrm{~kJ} \mathrm{~mol}^{-1}$ characterizing the $\sigma$ relaxation process. ${ }^{8}$

The deviation to higher temperatures of the location of this process in annealed samples relative to the wet ones is an indication that water as a plasticizing effect in this process.

The $\sigma$ process has been described has a diffusion/hopping process with the characteristics of a relaxation process, strongly correlated with dc-conductivity. Essentially two models attempt to molecularly interpret this $\sigma$ process: the "random potential model" by Stevels ${ }^{20}$ and Taylor ${ }^{21}$ based in specific mean distances between high potential barriers for the ion migration and the "conducting path model" by Yamamoto-Namikawa ${ }^{22}$ based in an inhomogeneous distribution of conducting pathways and nonconductive regions.

The fact that it is observed earlier in the temperature scale in nonneutralized chitosan, is an indication of the strong correlation with the conductivity, since this sample as abovementioned, has the onset of conductivity at lower temperatures when compared with the neutralized form due to either a proton enhanced mobility and/or the acetate counteranions.

This $\sigma$ process should not be interpreted as an internal interfacial relaxation since a separate MWS-process was additionally found at higher temperatures. In Figure 2, the increase in dielectric loss at the highest temperatures, mainly for nonneutralized annealed chitosan, was due to MWS relaxation. Figure 7 shows the MWS process at the lowfrequency side (indicated by the arrow) of the $\sigma$ process for a very well dried nonneutralized sample (this sample has been dried $48 \mathrm{~h}$ under vacuum at $140{ }^{\circ} \mathrm{C}$ and immediately annealed previously to the measurements).

\section{Concluding Remarks}

Chitosan presents essentially two arrhenian type dielectric relaxation processes, the first one located at temperatures lower than $0{ }^{\circ} \mathrm{C}$ (named process I) and the second one visible at temperatures higher than $0{ }^{\circ} \mathrm{C}$ (named process II).

In very well dried samples, a MWS process is visible at high temperatures in the low-frequency side of process II.
The low-temperature process (process I) is a $\beta$-type process that is ill defined in wet nonneutralized chitosan, being enhanced after water removal. This suggests an enhanced interaction of the $\mathrm{NH}_{3}{ }^{+}$side groups with water, strongly depleting process I. The differences in intensity in annealed samples lead us to conclude that the $\mathrm{NH}_{3}{ }^{+} / \mathrm{NH}_{2}$ side groups influence the local dynamics that is in the origin of the secondary relaxation process I.

After water removal, process I preserves its shape and location independently of the $\mathrm{NH}_{2} / \mathrm{NH}_{3}{ }^{+}$side group.

Process II was found to be compatible with the so-called $\sigma$ process, which is related with migration of conductive species. Its temperature location deviates to higher temperatures with the water removal, leading to the conclusion that water has a plasticizing effect in this process.

Process II is more mobile in nonneutralized chitosan which is an evidence of its strong correlation with the dc conductivity, since nonneutralized wet chitosan revealed both the onset and the maximum of conductivity at a lower temperature relative to the wet neutralized form. The nonneutralized sample reveals also higher conductivity values due to both higher mobility and number of conducting species $\left(\mathrm{H}^{+}\right.$and acetate anions).

Acknowledgment. This work was financially supported by FCT, Foundation for Science and Technology, through the POCTI (POCTI/CTM/47363/2002) and FEDER programs.

\section{References and Notes}

(1) Muzzarelli, R., Jeniaux, C., Gooday, G. W., Eds.; Chitin in Nature and Technology; Plenum Press: New York, 1985.

(2) Lazaridou, A. O.; Biliaderis, C. G. Carbohydr. Polym. 2002, 48, 179190

(3) Radloff, D.; Boeffel, C.; Spiess, H. W. Macromolecules 1996, 29, $1528-1534$.

(4) Montes, H.; Mazeau, K.; Cavaille, J. Y. Macromolecules 1997, 30, 6977-6984.

(5) Meissner, D.; Einfeldt, L.; Einfeldt, J. J. Polym. Sci., Phys. Ed. 2001, 39, 2491-2500.

(6) Butler, M. F.; Cameron, R. E. Polymer 2000, 41, 2249-2263.

(7) Einfeldt, J.; Maissner, D.; Kwasniewski, A.; Einfeldt, L. Polymer 2001, 42, 7049-7062

(8) Maissner, D.; Einfeldt, J.; Kwasniewski, A. J. Non-Cryst. Solids 2003, 320, 40-55.

(9) Einfeldt, J.; Maissner, D.; Kwasniewski, A. Prog. Polym. Sci. 2001, 26, 1419-1472.

(10) Nogales, A.; Ezquerra T. A.; Rueda, D. R.; Martínez, F.; Retuert, J. Colloid Polym. Sci. 1997, 275, 419-425.

(11) Baxter, A.; Dillon, M.; Taylor, KDA; Roberts, GAF. Int. J. Biol. Macromol. 1992, 14, 166-169.

(12) Brugnerotto, J.; Lizardi, J.; Goycoolea, F. M.; Arguelles-Monal, W.; Desbrieres, J.; Rinaudo, M. Polymer 2001, 42, 3569-3580.

(13) Terbojevich, M.; Cosani, A. Molecular weight determination of chitin and chitosan. In Chitin Handbook; Muzzarelli, R. A. A., Peter, M. G., Eds., European Chitin Society: Bremen, Gremany, 1997; pp 8893.

(14) Bégin, A.; Calsteren, M-R. V. Int. J. Biol. Macromol. 1999, $26,63$.

(15) Rinaudo, M.; Pavlov, G.; Desbrières, J. Polymer 1999, 40, 70297032

(16) Havriliak, S.; Negami, S. Polymer 1967, 8, 161.

(17) Goodford, P. J. J. Med. Chem. 1985, 28, 849-857.

(18) Wade, R. C.; Clark, K. J. A.; Goodford, P. J. J. Med. Chem. 1993 , 36, 140-147. Wade, R. C.; Goodford, P. J. J. Med. Chem. 1993, $36,148-156$.

(19) Eyring, H. J. Chem. Phys. 1936, 4, 183

(20) Stevels, J. M. J. Non-Crys. Solids 1980, 40, 69

(21) Taylor, H. E. Trans. Faraday Soc. 1956, 52, 873.

(22) Yamamoto, K.; Namikawa, H. Japan J. Appl. Phys. 1988, 27, 1845. BM049685B 